\title{
BMJ Open Mental health specialist video consultations for patients with depression or anxiety disorders in primary care: protocol for a randomised controlled feasibility trial
}

Justus Tönnies, ${ }^{1}$ Mechthild Hartmann, ${ }^{1}$ Michel Wensing, ${ }^{2}$ Joachim Szecsenyi, ${ }^{2}$ Andrea Icks, ${ }^{3,4}$ Hans-Christoph Friederich, ${ }^{1}$ Markus W Haun ${ }^{\odot 1}$

To cite: Tönnies J, Hartmann M, Wensing M, et al. Mental health specialist video consultations for patients with depression or anxiety disorders in primary care: protocol for a randomised controlled feasibility trial. BMJ Open 2019;9:e030003. doi:10.1136/ bmjopen-2019-030003

- Prepublication history and additional material for this paper are available online. To view these files, please visit the journal online (http://dx.doi. org/10.1136bmjopen-2019030003).

Received 22 February 2019 Revised 17 July 2019 Accepted 30 July 2019

\section{Check for updates}

(c) Author(s) (or their employer(s)) 2019. Re-use permitted under CC BY-NC. No commercial re-use. See rights and permissions. Published by BMJ.

For numbered affiliations see end of article.

Correspondence to Mr Markus W Haun; markus.haun@med.uniheidelberg.de

\section{ABSTRACT}

Introduction Most people suffering from depression and anxiety disorders are entirely treated in primary care. Due to growing challenges in ageing societies, for example, patients' immobility and multimorbidity, the transition to specialised care becomes increasingly difficult. Although the co-location of general practitioners and mental health specialists improves the access to psychosocial care, integrated in-person approaches are not practical for rural and single-doctor practices with limited personnel and financial resources. Treating primary care patients via internet-based video consultations by remotely located mental health specialists bears the potential to overcome structural barriers and provide low-threshold care. The aim of this randomised controlled feasibility trial is to investigate the feasibility of implementing of mental health specialist video consultations in primary care practices.

Methods and analysis Fifty primary care patients with significant depression and/or anxiety symptomatology will be randomised in two groups receiving either the treatment as usual as provided by their general practitioner or up to five video consultations conducted by a mental health specialist. The video consultations focus on (1) systematic diagnosis plus proactive monitoring using validated clinical rating scales, (2) the establishment of an effective working alliance and (3) a stepped-care algorithm within integrated care adjusting treatments based on clinical outcomes. We will investigate the following outcomes: effectiveness of the recruitment strategies, patient acceptance of randomisation, practicability of the technical and logistical processes related to implementing video consultations in the practices' workflows, feasibility of the data collection and clinical parameters.

Ethics and dissemination This trial has undergone ethical scrutiny and has been approved by the Medical Faculty of the University of Heidelberg Ethics Committee (S-634/2018). The findings will be disseminated to the research community through presentations at conferences and publications in scientific journals. This feasibility trial will prepare the ground for a large-scale, fully powered randomised controlled trial.

Trial registration number DRKS00015812.

\section{Strengths and limitations of this study}

- This is one of the first feasibility trials investigating mental health specialist video consultations for patients presenting in primary care.

- While gathering comprehensive multimodal data on feasibility outcomes, we will also longitudinally assess the development of the conversation quality and the therapeutic alliance in the course of the video consultations.

- Given the nature of our feasibility study, we will not be able to determine intervention effectiveness at this point, but our findings will inform the design of a sufficiently powered randomised controlled trial.

\section{INTRODUCTION}

Depression and anxiety disorders are highly prevalent, cause a substantial disease burden and account for the two highest numbers of disability-adjusted life years among all mental disorders. ${ }^{12}$ Most patients with depression and anxiety disorders are treated in primary care, which has evolved as the crucial point of mental healthcare in many high-income countries. ${ }^{3}$ However, while general practitioners (GPs) generally provide comprehensive care to most of their patients, a significant number of people, especially those with somatic comorbidities, suffering from mental health conditions do not get adequate treatment. ${ }^{4}$ This problem is aggravated by patient-related and system-related determinants. The access to specialised mental healthcare is impeded by long waiting times at the provider's end along with older patients' immobility due to increasing multimorbidity in an ageing society. ${ }^{5}$ Multimorbidity in turn constitutes a challenge for GPs who are often obligated to emphasise the assessment and treatment of somatic symptoms due to guideline 
recommendations. ${ }^{6}$ However, for GPs, prioritising somatic work-up and treatment conflicts with devoting their time and attention to psychosocial aspects. ${ }^{7}$

To resolve these challenges, it is essential to develop healthcare models that combine the easily accessible environment of primary care and the expertise in timely diagnostics and therapy of a mental health specialist (MHS). These models may help providing a tailored treatment for patients initially presenting to their GP. Indeed, two principal approaches for fostering the co-operation and co-ordination between GPs and MHS have evolved. In the Collaborative Care (CC) model, the GP is supported by a case manager who tracks patients per telephone, conducts psychological assessments and presents the data to an MHS, often a psychiatrist. The MHS monitors the patients and intervenes, if necessary, by prescribing drugs or scheduling face-to-face contacts. This time-saving model reaches a higher number of patients in specialised mental care than the usual referral-based system as the MHS is not required to see all patients regularly. ${ }^{8}$ The Primary Care Behavioural Health (PCBH) model co-locates the primary care team and the MHS. ${ }^{9}{ }^{10}$ Specifically, the MHS provides "high volume services that are accessible, team-based, and a routine part of primary care". ${ }^{10}$ For example, at Cherokee Health Systems, a complete community health system in Tennessee in the USA (https://www.cherokeehealth.com/), MHS engage in goal setting together with patients, in patient activation and in psychosocial care. ${ }^{11}$ Often MHS attend to patients through warm hand-offs instead of receiving conventional referrals. This model provides a low-threshold access to specialised care and has been well accepted in patients and providers.

Both CC and PCBH have been implemented successfully in the US healthcare system. ${ }^{12}$ However, even in the USA, where the mean number of primary care physicians per practice is much higher than in European countries, such as the UK, France and Germany, small and remote practices especially in rural areas often do not have the resources to employ an additional MHS. ${ }^{13}$ Consequently, these barriers are even higher in healthcare systems where smaller practices (with one or two doctors) with rather limited resources predominate. It is crucial to investigate potentially more feasible modes for putting these integrated care models into practice.

As an innovative, technology-supported approach to integrated care, real-time video consultations conducted by MHS are increasingly considered to be an alternative to in-person settings. Systematic reviews point to the general effectiveness of telemedicine interventions for mental health conditions. ${ }^{14-16}$ Concerning the integration of telepsychiatry services in primary care as such, several observational and interventional studies demonstrate that mental health specialist video consultations (MHSVCs) contribute to overcoming geographical barriers and to treating the increasing number of multimorbid patients often cut off from specialised care. ${ }^{17-20}$ However, the randomised controlled trials (RCTs) were conducted in selected healthcare settings (US Veterans Healthcare Administration, Rural Federally Qualified Health Centers) and therefore their results are of limited generalisability, for example, to European healthcare systems. ${ }^{17} 18$ Nevertheless, patients may benefit from timely access to specialised care, less stigmatisation, more personal proximity compared with self-help and telephone interventions, and better compatibility with work-related or domestic obligations in the light of reduced travel times. ${ }^{21}$

The junior research group PROVIDE (ImPROving cross-sectoral collaboration between primary and psychosocial care: An implementation study on VIDEo consultations) aims to define, tailor and evaluate an integrated care model compatible with small and/or remote GP offices where the patient will receive the telemedical service. In contrast, the MHS will be situated in her or his office/private practice or a suitable, designated room at home. The project is funded by the German Ministry of Education and Research and culminates in a largescale RCT. In this protocol, we describe a randomised controlled feasibility trial, which will assess the feasibility of research methods and the intervention: mental health specialist video consultations in primary care.

\section{METHODS AND ANALYSIS \\ Study design}

This study is a multicentric, prospective, assessor blinded and individually randomised controlled feasibility trial. After inclusion of patients, the individual intervention period will be 3 months; the total time of recruitment is planned to be 6 months. There will be two measurements including a baseline assessment just prior to randomisation and post-assessment at 4 months after inclusion. The study will be implemented and reported in line with Standard Protocol Items: Recommendations for Interventional Trials (SPIRIT) guidelines ${ }^{22}$ (online supplementary appendix 1).

\section{Sample size}

We project a target sample size of 50 participants. Since the aim of this study is to establish feasibility of a full trial, but no detection of statistically significant difference in efficacy between the arms, a formal sample size calculation was not carried out. A sample size of 50 patients offers a reasonable test of the intervention to assess the feasibility objectives. This sample size is also recommended for pilot and feasibility trials by The National Institute for Health Research. ${ }^{23} 24$

\section{Recruitment}

\section{General practitioners}

In this feasibility study, four family practices will be recruited. Each practice then will recruit approximately 13 patients. Access to them will be sought first by contacting GPs who participated in a preceding qualitative pre-implementation study and second through the network of 
collaborating academic research practices affiliated with the Department of General Practice and Health Services Research at Heidelberg University. During on-site visits, we will inform the practice teams about the study including the concomitant process evaluation and the assessments involved. We will also test the quality of the internet connexion to evaluate the eligibility. The participation requires the signed informed consent.

\section{Mental health specialists}

Recruitment of the therapists will be conducted at the Institute for Psychotherapy, Heidelberg (HIP), which is a state-approved psychotherapeutic training facility at Heidelberg University Hospital. We will recruit four psychotherapists or psychotherapy trainees, that is, psychologists in the advanced training period, in total, one for each general practice. We will contact all lecturers (mostly licensed psychotherapists) and trainees systematically using the respective mailing lists. Interested individuals will have to apply and will then be invited for a short interview. The final decisions on the participating psychotherapists will be made by mutual discussion and consensus formation in the study team. The MHS will participate in the study as freelancers and will be paid per session according to the current fees for psychotherapy as reimbursed by the German statutory health insurance. For the therapists, expected time expenditure will be approximately 6 hours per week (4hours for consultations, 1.5 hours for supervision).

\section{Patients}

GPs will recruit patients during their regular clinic hours. Based on their clinical judgement, GPs will prospectively select individuals suspected to suffer from depression or anxiety and present the study to them by offering information material. If the patient agrees to receive more information, she or he will be contacted by the study team who will screen her or him with respect to the eligibility criteria. We will send a hard-copy version of the study information booklet and the informed consent form to all the patients interested in participation. At this point, patients will be able to raise questions with the principal investigator. Study participation requires a signed informed consent which the patients will mail back to the trial co-ordination centre. Whenever inclusion is not possible, we will record the reason, the recruiting general practice along with patient age and gender.

\section{Inclusion and exclusion criteria \\ Patients}

Inclusion criteria require patients to (1) exceed cut-offs of 9 points (a) for the Patient Health Questionnaire (PHQ-9) and/or (b) for the Generalized Anxiety Disorder 7 (GAD-7), ${ }^{25}$ respectively, (2) currently have no or as yet insufficient treatment (psychotherapy, psychopharmacotherapy or both) or difficulty with adherence, (3) agree to participate in the study by written informed consent, (4) be capable of giving consent and (5) be
18 years or older. Exclusion criteria are as follows: (1) substance abuse/dependence that is likely to compromise intervention adherence; (2) risk of endangerment to others and/or risk of self-endangerment; (3) need for emergency medical treatment, for example, admission; (4) acute psychotic symptoms, for example, persecutory delusions and/or thought insertion; (5) severe cognitive impairment or dementia; (6) significant hearing and/or visual impairment; (7) pregnancy in the $\geq 2$ nd trimester; (8) insufficient German language proficiency.

\section{Primary care practices and mental health specialists}

Inclusion criteria for practices are as follows: (1) primary practice (specialist in general medicine or internal medicine), (2) team that familiarises patients with video consultations and (3) written informed consent. Exclusion criteria for the practices are lack of a designated room for the video consultations to ensure confidentiality and lack of internet access or low bandwidth ( $<384 \mathrm{kbps})$.

Participating MHS must be licensed psychotherapists or advanced trainee in psychotherapy (that is, at least 1200 hours of treatment experience) and give written informed consent.

\section{Randomisation}

Patients will be randomly allocated to one of the two study conditions (video consultation model vs treatment-asusual) in a 1:1 ratio. After having obtained the informed consent, the randomisation will be conducted centrally at the study centre. The web-based application Randomizer V.2.0.2 of the Institute for Medical Informatics, Statistics and Documentation of the Medical University of Graz, Austria (https://www.randomizer.at) will be used, which ensures the concealment of the treatment sequence up to the allocation by central randomisation. The treatment sequence is generated through a computer-generated sequence of random numbers. We will apply block randomisation with stratification of patients by office. A member of the Institute of Medical Biometry and Informatics, Heidelberg University, not involved in the patient recruitment will randomise. We will ensure that the assessors who will collect the data will be blinded to the allocated treatment.

\section{Study procedures}

During an introductory training, we will familiarise the therapists and supervisors participating in the study and cover (1) an introduction to the research project, (2) a detailed description of the study procedures, (3) a stepby-step instruction on the handling of video consultations (eg, room setup and technical aspects), (4) an introduction to the intervention and (5) a familiarisation with the respective general practice. ${ }^{26-30}$ For enrolling and preparing practices, study team members will visit each practice twice. During the first visit, we will check for a designated room suitable for confidential video consultations, enough bandwidth and technical equipment. If the practice is eligible, we will conduct a second visit during 
which we will (1) explain the study procedures in greater detail, (2) obtain informed consent from the practice team, (3) introduce the assigned therapist personally, (4) prepare the technical equipment, (5) train the GP in expense presenting the study to patients (eg, by dispensing jointly designed brochures and positioning waiting room posters both tailored to the respective practice) and (6) clarify the regular time slots for the consultations. We will train all clinicians in initiating the consultations logistically and technically drawing on the latest guidance available. ${ }^{27}$ To mitigate potential insecurities, we will also inform the clinicians that there is currently "[...] no evidence to suggest prohibiting trainees or clinicians from engaging in telehealth if they are otherwise qualified". ${ }^{31}$ We will ensure that every practice will nominate one team member who will be responsible for initiating video consultations and who will serve as contact person for MHS, patients and the study team. Applying a training that primarily targets technical competency, we are confident that we will minimise potential difficulties with handling video consultations and consequently minimise task-related expenses. In fact, technical competency is regarded as crucial for successfully implementing telepsychiatry services. ${ }^{32}$ Additionally, we will address remaining questions and equip the teams with a study handbook including description of the secure videoconferencing platform and contingency plans in case of technical failures. GPs will then start recruiting patients by forwarding their contact information to the study team. To screen patients using the PHQ-9 and GAD-7, the study team will conduct standardised computer-assisted telephone interviews. After providing written informed consent, patients will fill out the baseline questionnaire and mail it back to

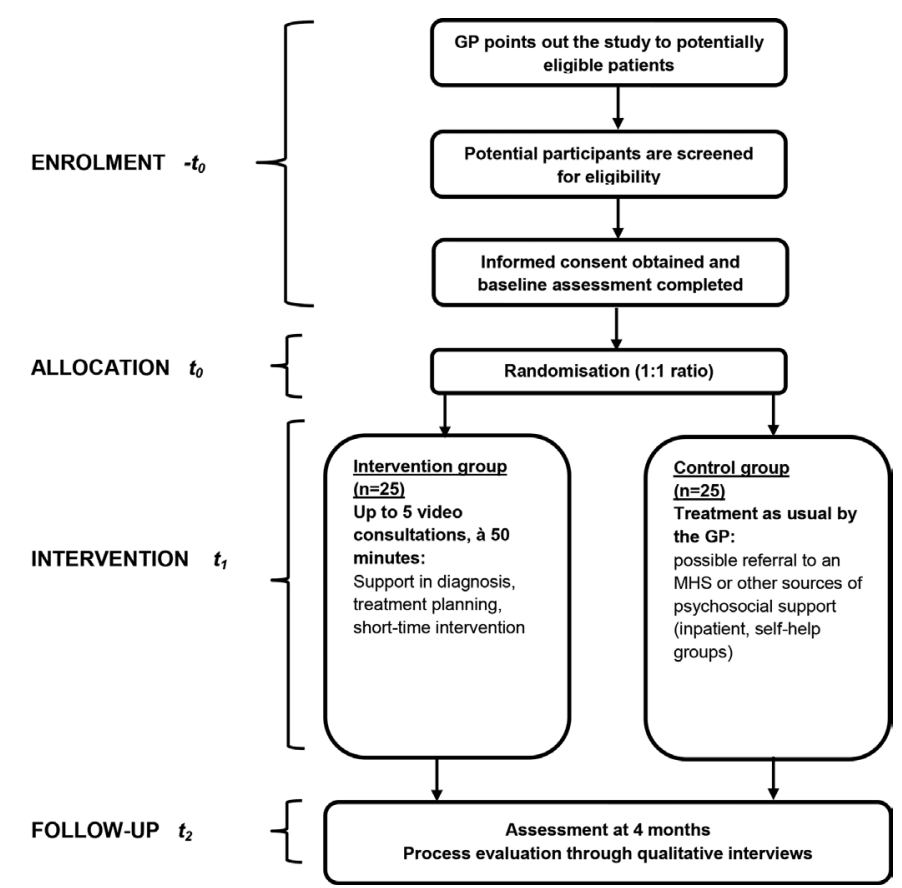

Figure 1 Study flowchart. GP, general practitioner; MHS, mental health specialist. the trial co-ordination centre. Patients will be randomly allocated to the intervention or the control condition. The study flow is depicted in figure 1 .

\section{Intervention}

The PROVIDE intervention is a targeted primary carebased mental health service that combines elements of the collaborative care and consultation-liaison model. Specifically, the intervention features web-based, realtime video consultations involving a live two-way interactive video to a primary care practice between MHS and patients. The intervention includes three core processes ('active ingredients') for effective primary care-based mental healthcare, namely systematic diagnosis plus proactive monitoring using validated clinical rating scales, the establishment of an effective working alliance and a stepped-care algorithm within integrated care adjusting treatments based on clinical outcomes. If indicated, the PROVIDE intervention also includes brief psychological therapy that works with interpersonal dynamics and that has been shown to confer additional benefit. ${ }^{33}$ The intervention follows a transdiagnostic treatment approach for emotional disorders(depression and anxiety), for which various meta-analyses have shown the efficacy compared with control conditions on measures of overall anxiety, disorder-specific anxiety and depression. ${ }^{34} 35$ Specifically, the intervention combines elements from problem-solving therapy, which has been shown to yield moderate effects in alleviating depression and anxiety in primary care. ${ }^{36}$ Psychodynamic elements following a relationship focus and interpersonal understanding are added to foster the working alliance that has been promoted as a crucial element of manuals achieving high acceptability in both patient and clinicians. ${ }^{37}$ In line with a stage model of psychotherapy manual development, ${ }^{38}$ we compiled a stage I intervention manual delineating treatment techniques, goals and format (supplementary appendix 2). For a description of the intervention, we followed the TIDieR checklist (supplementary appendix 3$).{ }^{39}$

Patients will receive their first video consultation shortly after randomisation and will be scheduled for up to five sessions, lasting $50 \mathrm{~min}$ each, in biweekly intervals. The video consultations will be carried out on a secure (ie, encrypted), web-based videoconferencing platform on a subscription basis (arztkonsultation ak $\mathrm{GmbH}$, https://arztkonsultation.de) at the fixed time slots set by the general practice staff. The patient will be in the general practice and the psychotherapist either in her or his office/private practice, in a suitable, designated room at home or in a therapy room at the HIP. Each MHS is permanently assigned to one general practice. At the beginning of each consultation, a practice team member will escort the patient to the room designated for video consultations, set up the computer tablet and the videoconferencing platform, if applicable, address the patient's questions and then leave the room. After the third session, we will conduct an interim evaluation of the 
symptoms (using PHQ-9 and GAD-7) and feedback these results to the therapist. After the last consultation with the patient, the MHS will send a written case summary to the GP which will be attached to the medical record in the primary care practice and on which, if needed, further decisions on follow-up procedures between GPs and MHS can be based.

The study therapists, psychologists with a diploma or master's degree or medical doctors, will be required to be trained in psychodynamic psychotherapy or cognitive-behavioural therapy for at least 3 years. Parallel to the study, they will receive weekly group supervision led by a senior consultant both in psychiatry and psychosomatic medicine from the Department of General Practice and Psychosomatics, Heidelberg University.

\section{Control condition}

Patients allocated to the control group will get the usual care provided by the GP. This may or may not include a referral to an MHS. We expect that most people with depression and/or anxiety disorders are currently treated by their GP only. GPs rather provide brief counselling and prescribe psychotropic medication than conduct psychotherapy as laid out in guidelines. Only every fifth patient with depression is referred to specialised care. ${ }^{5}$ There will not be any restrictions to the usual treatment by the GP.

\section{Measurements}

Patients' health status

For patients, the baseline assessment will take place before randomisation and include a set of validated questionnaires: PHQ- $9,{ }^{25}$ GAD-7, ${ }^{12}$ German version of the Recovery Assessment Scale ${ }^{40}$ EQ-5D,${ }^{41}$ Somatic Symptom Disorder-B Criteria Scale ${ }^{42}$ and Patient Assessment of Chronic Illness Care. ${ }^{43}$ Following the Medical Research Council guidance on developing and evaluating complex interventions, ${ }^{44}$ a health-economic evaluation is planned for the main trial. Hence, a questionnaire on the use of services is part of the assessment instruments. ${ }^{45}$ Post-measurements will be scheduled 4 months after inclusion in the study and will include the same measures as well as the Inventory for the Assessment of Negative Effects of Psychotherapy for measuring potential adverse effects. ${ }^{46}$ As part of the blind outcome assessment, two research assistants, blinded to participant allocation, will conduct the post-measurement in telephone interviews with the participants. In line with current recommendations, we specifically will make sure that the outcome assessors will not be present when discussing individual patients and avoid mentioning any names or assigned treatments. ${ }^{47}$ In addition, we will instruct patients before the interview not to mention which group, control or intervention, they belonged to. In the case of unintentional unblinding during the assessment, the assessors will document how and at which point the unblinding unfolded. Hence, we will be able to subsequently determine the extent to which blinded assessment was successful. The study schedule is depicted in figure 2 in line with the SPIRIT guidelines.

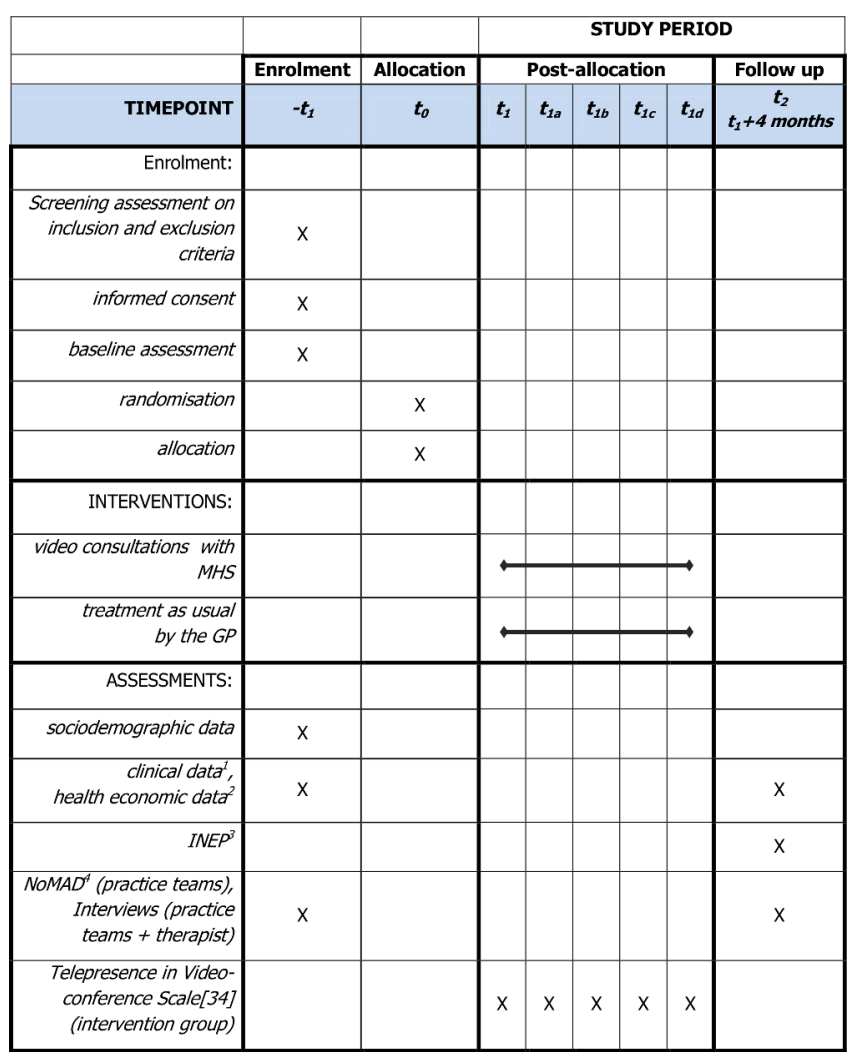

Figure 2 Study schedule. ${ }^{22}$ 1. Patient Health Questionnaire 9 (PHQ-9), ${ }^{25}$ Generalised Anxiety Disorder Scale (GAD7), ${ }^{25}$ Recovery Assessment Scale (RAS-G), ${ }^{40} \mathrm{EQ}-5 \mathrm{D},{ }^{41}$ Somatic Symptom Disorder-B Criteria Scale (SSD-12). ${ }^{42}$

2. Questionnaire for the Assessment of Medical and non-Medical Resource Utilisation in Mental Disorders (FIMPsy). ${ }^{45}$ 3. Inventory for the Assessment of Negative Effects of Psychotherapy (INEP). ${ }^{46}$ 4. Normalisation MeAsure Development (NoMAD) questionnaire. ${ }^{49} \mathrm{GP}$, general practitioner; MHS, mental health specialist.

\section{Feasibility}

To assess process feasibility from the patients' perspective, we will conduct semi-guided qualitative interviews with 10 purposefully selected patients from the intervention group stratified by practice site, patient age and patient technology commitment. By applying this criterion, we aim for maximising the transferability of our feasibility findings, for example, with respect to compatibility with existing clinical workflows. To evaluate the acceptability of both study and intervention procedures, we will analyse patients' perceptions whether the intervention and study procedures have been agreeable as well as logistically and technically practical. ${ }^{48}$

As part of a pre-post-study measurement of feasibility, all practice teams and MHS will fill out the Normalisation MeAsure Development (NoMAD) questionnaire ${ }^{49}$ prior to inclusion of the first patient and close-out of the last patient. This questionnaire assesses providers' and staffs' perceptions of factors relevant to embedding interventions that change their work practices. Furthermore, as part of the post-measurement, all GPs, all team members responsible for initiating video consultations and all MHS 
will participate in individual qualitative semiguided interviews to describe feasibility, adequacy and acceptance of the proposed model in greater detail. To estimate the conversation quality of the video consultations, patients and MHS will complete the 8-item Telepresence in Videoconference Scale at the end of each consultation. ${ }^{50}$ This instrument assesses the subjective feeling of presence in psychotherapy accounting for the three factors physical presence, social presence and absorption. From these high-frequency repeated measurements, we aim to document the level of stability in the quality of the consultations (eg, habituation effects, ruptures during the intervention).

\section{Outcomes}

To determine the feasibility of a subsequent large-scale RCT, we will assess the following outcomes and aspects:

- Sufficiency and efficiency of recruitment strategies for intervention and control group

- Recruitment and attrition rates for intervention and control group

- Patient and provider acceptance of outcome measurements.

- Feasibility of study procedures (eg, patient and provider acceptance of randomisation and outcome measurements).

- Feasibility of intervention procedures (including the necessary documentation, implementation loyalty, patient acceptance of video consultations, patient safety).

We will operationalise the sufficiency of recruiting strategies and the acceptance of randomisation and by measuring recruitment and retention rates, including recruitment patterns from each route and geographical area. We will ask patients declining to participate or dropping out to provide their reasons for doing so. With respect to process outcomes on the overall practicability of the intervention and the related study procedures, we will draw on qualitative data generated by in-depth interviewing of patients, practice staff and MHS.

\section{Data analysis}

To promote data quality, data management procedures will include double data entry and a comprehensive plausibility check (eg, concerning range of data values). Only the first and the corresponding author will have access to the final trial dataset. Quantitative data regarding the feasibility of a following large-scale RCT, for example, recruitment rates and attrition at study completion, along with health outcomes from questionnaires will be analysed applying descriptive statistics (absolute and relative frequencies, measures of central tendency and measures of variability). Specifically, the number of potential participants contacted, screened and included will be reported. The percentages of potential participants willing to undergo screening assessment of the total number invited and of participants overall enrolled in the study out of the total number invited will be calculated with $95 \%$ CIs.
To illustrate participant flow, we will report results in a CONSORT diagram. We will describe patients' reasons for non-participation and numbers of enrolled practices by recruitment strategy. We will also analyse questionnaire outcome data (including NoMAD) descriptively. Qualitative data generated in the process evaluation will be subjected to thematic analysis which we will conduct in the qualitative data analysis software MAXQDA. Specifically, we will derive key theme bottom-up. We will align all study publications with recommendations from statements for observational and feasibility studies.

\section{Patient and public involvement}

This protocol was drafted without patient involvement. Patients were not consulted to develop patient-relevant outcomes or interpret the results. Patients were not invited to contribute to the writing or editing of this document for readability or accuracy. However, we did conduct focus groups with patient, MHS, GPs and medical assistants in primary care practices along with health policy-makers as part of pre-implementation study. ${ }^{51}{ }^{52}$ Results from these focus groups significantly impacted on tailoring the intervention and the study procedures. We analysed and interpreted the results within two in-depth discussions within the study team. Main aspects and suggestions have been incorporated into the handbook for GPs and the study manual for MHS that provide guidance regarding the study procedures and the intervention. Examples which have been transferred from focus groups' results are that (1) the appointment management were put into the hands of the MHS, (2) fixed time slots will be used and (3) each patient will continuously consult with the identical MHS.

\section{ETHICS AND DISSEMINATION}

Participants will be asked to provide informed consent prior to baseline assessment. In advance, they will receive detailed information about the study and their right to withdraw it without the obligation to give reasons. To adequately prepare for the emerging field of telemedicine, the study manual for MHS will be based on existing recommendations for telepsychiatry. ${ }^{26}{ }^{28-30}$ Moreover, MHS will be supported by a weekly supervision which will be led by a senior consultant both in psychiatry and psychosomatic medicine from the Department of General Internal Medicine and Psychosomatics, Heidelberg University. This will help to react to unintended effects during the study. We do not expect major relevant risks for participants irrespective of phases of emotional arousal which frequently occur during psychotherapy. The time burden for the participants arising from the assessments will be of a reasonable amount. The data collection and storage will be conducted in accordance with the General Data Protection Regulation which ensures a high level of data safety and a conscientious handling of all the patient, practice and therapist data. Additionally, considering that the study will take place in routine general practice, we obtained the ethical approval 
of the State Chamber of Physicians Baden-Württemberg. As part of a wider dissemination, the results of this feasibility trial will inform the setup of a large-scale randomised trial which is supposed to evaluate broad regional implementation of MHSVC in primary care. Moreover, we will publish and present key outputs on conferences and in internationally recognised peer-reviewed journals.

\section{DISCUSSION}

To the best of our knowledge, we conduct one of the first feasibility trials on mental health specialist video consultations for patients with depression or anxiety disorders in European routine primary care. In more specific contexts, particularly in the USA, facilitating the integration of primary care and mental healthcare through video consultations has been shown to potentially overcome geographical and temporal barriers in the search for specialised mental healthcare. As a low-threshold model embedded in a familiar environment, MHSVC in primary care may be of particular value for patients who are currently comparatively cut off from specialised care. This trial will deliver results on the feasibility of this model and prepare the ground for a fully powered RCT on its broader roll-out.

\section{Author affiliations \\ ${ }^{1}$ Department of General Internal Medicine and Psychosomatics, Heidelberg University, Heidelberg, Germany \\ ${ }^{2}$ Department of General Practice and Health Services Research, Heidelberg University, Heidelberg, Germany \\ ${ }^{3}$ Institute of Health Services Research and Health Economics, Heinrich Heine University, Düsseldorf, Germany \\ ${ }^{4}$ Institute for Health Services Research and Health Economics, German Diabetes Center (DDZ), Leibniz Institute for Diabetes Research at Heinrich Heine University Düsseldorf, Düsseldorf, Germany}

Contributors All authors contributed substantially to the conception and the design of the study. JT, MH, MW, JS, Al, and MWH contributed to data analysis plan. JT, MH, HC-F, and MWH drafted the manuscript. All authors including MW, Al, and $\mathrm{HC}-\mathrm{F}$ revised the manuscript critically for important intellectual content. All authors approved the version of the manuscript to be published and have agreed to be accountable for all aspects of the work

Funding This work was supported entirely by the German Federal Ministry of Education and Research (BMBF) (grant no. 01GY16129). The funding agreement ensured the authors' independence in designing the study, interpreting the data, writing and publishing the report.

Competing interests None declared.

Patient consent for publication Not required.

Provenance and peer review Not commissioned; externally peer reviewed.

Open access This is an open access article distributed in accordance with the Creative Commons Attribution Non Commercial (CC BY-NC 4.0) license, which permits others to distribute, remix, adapt, build upon this work non-commercially, and license their derivative works on different terms, provided the original work is properly cited, appropriate credit is given, any changes made indicated, and the use is non-commercial. See: http://creativecommons.org/licenses/by-nc/4.0/.

\section{REFERENCES}

1. Whiteford HA, Degenhardt L, Rehm J, et al. Global burden of disease attributable to mental and substance use disorders: findings from the Global Burden of Disease Study 2010. Lancet 2013;382:1575-86.
2. Whiteford HA, Ferrari AJ, Degenhardt L, et al. The global burden of mental, neurological and substance use disorders: an analysis from the Global Burden of Disease Study 2010. PLoS One 2015;10:e0116820.

3. Linde K, Sigterman K, Kriston L, et al. Effectiveness of psychological treatments for depressive disorders in primary care: systematic review and meta-analysis. Ann Fam Med 2015;13:56-68.

4. Trautmann S, Beesdo-Baum K. The treatment of depression in primary care. Deutsches Ärzteblatt International 2017;114:721-8.

5. Cunningham PJ. Beyond parity: primary care physicians' perspectives on access to mental health care. Health Aff 2009;28(Supplement 1):w490-501.

6. Cassano P, Fava M. Depression and public health: an overview. $J$ Psychosom Res 2002;53:849-57.

7. Moussavi S, Chatterji S, Verdes E, et al. Depression, chronic diseases, and decrements in health: results from the World Health Surveys. Lancet 2007;370:851-8.

8. Unützer Jet al. The Collaborative Care Model: an approach for integrating physical and mental health care in Medicaid home, Health Home Information Resource Center. Editor 2013.

9. Crowley RA, Kirschner N. The integration of care for mental health, substance abuse, and other behavioral health conditions into primary care: executive summary of an American College of Physicians position paper. Ann Intern Med 2015;163:298-9.

10. Reiter JT, Dobmeyer AC, Hunter CL. The primary care behavioral health (PCBH) model: an overview and operational definition. J Clin Psychol Med Settings 2018;25:109-26.

11. Kanapaux W. The road to integrated care: commitment is the key. Tennessee CMHC demonstrates promise of co-located behavioral and primary care. Behav Healthc Tomorrow 2004;13:10-15.

12. Sandoval BE, Bell J, Khatri P, et al. Toward a unified integration approach: uniting diverse primary care strategies under the primary care behavioral health (PCBH) model. J Clin Psychol Med Settings 2018;25:187-96.

13. Schoen C, Osborn R, Doty MM, et al. A survey of primary care physicians in eleven countries, 2009: perspectives on care, costs, and experiences. Health Aff 2009;28(Supplement 1):w1171-83.

14. Bashshur RLet al. The empirical evidence for telemedicine interventions in mental disorders. Telemedicine and e-Health 2015;22:1-27.

15. Hilty DM, Rabinowitz T, McCarron RM, et al. An update on telepsychiatry and how it can leverage collaborative, stepped, and integrated services to primary care. Psychosomatics 2018;59:227-50.

16. Shore $\mathrm{JH}$. Telepsychiatry: videoconferencing in the delivery of psychiatric care. Am J Psychiatry 2013;170:256-62.

17. Fortney JC, Pyne JM, Edlund MJ, et al. A randomized trial of telemedicine-based collaborative care for depression. J Gen Intern Med 2007;22:1086-93

18. Fortney JC, Pyne JM, Mouden SB, et al. Practice-based versus telemedicine-based collaborative care for depression in rural federally qualified health centers: a pragmatic randomized comparative effectiveness trial. Am J Psychiatry 2013;170:414-25.

19. Fortney JC, Pyne JM, Turner EE, et al. Telepsychiatry integration of mental health services into rural primary care settings. Int Rev Psychiatry 2015;27:525-39.

20. Shore J. The evolution and history of telepsychiatry and its impact on psychiatric care: current implications for psychiatrists and psychiatric organizations. Int Rev Psychiatry 2015;27:469-75.

21. Yellowlees P, Richard Chan S, Burke Parish M. The hybrid doctorpatient relationship in the age of technology - Telepsychiatry consultations and the use of virtual space. Int Rev Psychiatry 2015;27:476-89.

22. Chan A-W, Tetzlaff JM, Gøtzsche PC, et al. Spirit 2013 explanation and elaboration: guidance for protocols of clinical trials. BMJ 2013;346:e7586.

23. National Institute for Health Research (NIHR). Justifying sample size for a feasibility study (online). Available: https://www.rds-london.nihr. ac.uk/RDSLondon/media/RDSContent/files/PDFs/Justifying-SampleSize-for-a-Feasibility-Study_1.pdf [Accessed cited 6 Jun 2019].

24. Sim J, Lewis M. The size of a pilot study for a clinical trial should be calculated in relation to considerations of precision and efficiency. $J$ Clin Epidemiol 2012;65:301-8.

25. Kroenke K, Spitzer RL, Williams JBW, et al. The patient health questionnaire somatic, anxiety, and depressive symptom scales: a systematic review. Gen Hosp Psychiatry 2010;32:345-59.

26. Joint Task Force for the Development of Telepsychology Guidelines for Psychologists, Guidelines for the practice of telepsychology. American Psychologist 2013;68:791-800.

27. Shore $\mathrm{JH}$, Yellowlees P, Caudill R, et al. Best practices in videoconferencing-based telemental health April 2018. Telemed J E Health 2018;24:827-32. 
28. Tuerk PW, Shore P. Clinical videoconferencing in telehealth-program development and practice. In: Tuerk PW, Shore P, eds. Behavioural telehealth. Cham, Heidelberg, New York, Dordrecht, London: Springer, 2015

29. Turvey C, Coleman M, Dennison O, et al. Practice guidelines for video-based online mental health services. . American Telemedicine Association, 2013: 19. 722-30.

30. Yellowlees P, Shore JH. Telepsychiatry and health technologies: a guide for mental health professionals. Arlington, VA: American Psychiatric Association Publishing, 2018.

31. Sanchez Gonzalez MLet al. Telemental health training and delivery in primary care: a case report of interdisciplinary treatment. J Clin Psychol 2019;75:260-70.

32. Hilty DM, Sunderji N, Suo S, et al. Telepsychiatry and other technologies for integrated care: evidence base, best practice models and competencies. Int Rev Psychiatry 2018;30:292-309.

33. Coventry PA, Hudson JL, Kontopantelis E, et al. Characteristics of effective collaborative care for treatment of depression: a systematic review and meta-regression of 74 randomised controlled trials. PLoS One 2014;9:e108114.

34. Craske MG. Transdiagnostic treatment for anxiety and depression. Depress Anxiety 2012;29:749-53.

35. Newby JM, McKinnon A, Kuyken W, et al. Systematic review and meta-analysis of transdiagnostic psychological treatments for anxiety and depressive disorders in adulthood. Clin Psychol Rev 2015;40:91-110.

36. Zhang A, Franklin C, Jing S, et al. The effectiveness of four empirically supported psychotherapies for primary care depression and anxiety: a systematic review and meta-analysis. J Affect Disord 2019;245:1168-86.

37. Fonagy P. Achieving evidence-based psychotherapy practice: a psychodynamic perspective on the general acceptance of treatment manuals. Clin Psychol 1999;6:442-4.

38. Carroll KM, Nuro KF. One size cannot fit all: a stage model for psychotherapy manual development. Clin Psychol 2002;9:396-406.

39. Hoffmann TC, Glasziou PP, Boutron I, et al. Better reporting of interventions: template for intervention description and replication (TIDieR) checklist and guide. BMJ 2014;348:g1687.

40. Cavelti M, Wirtz M, Corrigan $P$, et al. Recovery assessment scale: examining the factor structure of the German version (RAS-G) in people with schizophrenia spectrum disorders. Eur Psychiatry 2017;41:60-7.

41. Greiner W, Claes C, Busschbach JJV, et al. Validating the EQ-5D with time trade off for the German population. Eur $J$ Health Econ 2005;6:124-30.
42. Toussaint A, Murray AM, Voigt K, et al. Development and validation of the Somatic Symptom Disorder-B Criteria Scale (SSD-12). Psychosom Med 2016;78:5-12.

43. Glasgow RE, Whitesides $\mathrm{H}$, Nelson $\mathrm{CC}$, et al. Use of the patient assessment of chronic illness care (PACIC) with diabetic patients: relationship to patient characteristics, receipt of care, and selfmanagement. Diabetes Care 2005;28:2655-61.

44. Craig P, Dieppe P, Macintyre S, et al. Developing and evaluating complex interventions: the new Medical Research Council guidance. BMJ 2008;337.

45. Grupp H, König H-H, Riedel-Heller S, et al. [FIMPsy-Questionnaire for the Assessment of Medical and non Medical Resource Utilisation in Mental Disorders: Development and Application]. Psychiatr Prax 2018;45:87-94.

46. Ladwig I, Rief W, Nestoriuc Y. Welche Risiken und Nebenwirkungen hat Psychotherapie? - Entwicklung des Inventars zur Erfassung Negativer Effekte von Psychotherapie (INEP). Verhaltenstherapie 2014;24:252-63.

47. Minns Lowe CJ, Wilson MS, Sackley CM, et al. Blind outcome assessment: the development and use of procedures to maintain and describe blinding in a pragmatic physiotherapy rehabilitation trial. Clin Rehabil 2011;25:264-74.

48. Brownson RC, Colditz GA, Proctor EK. Dissemination and implementation research in health: translating science to practice. Oxford University Press, 2018.

49. Finch TL, Girling M, May CR, et al. Improving the normalization of complex interventions: part 2-validation of the NoMAD instrument for assessing implementation work based on normalization process theory (NPT). BMC Med Res Methodol 2018;18:135.

50. Bouchard Set al. Presence and the bond between patients and their psychotherapists in the cognitive-behavior therapy of panic disorder with agoraphobia delivered in videoconference. In Proceedings of the 10th Annual International Workshop on Presence, Barcelona, 2007.

51. Hoffmann M, Hartmann M, Wensing $M$, et al. Potential for integrating mental health specialist video consultations in office-based routine primary care: cross-sectional qualitative study among family physicians. J Med Internet Res 2019;21:e13382.

52. Haun MW, Tönnies J, Oeljeklaus L, et al. Psychotherapeutic video consultations in primary care - concept of the PROVIDE intervention and results on the intent to adopt in psychotherapists. Psychotherapie - Psychosomatik - Medizinische Psychologie 2019;69:1-4. 